\title{
Validation of 34betaE12 immunoexpression in clear cell papillary renal cell carcinoma as a sensitive biomarker
}

\section{Martignoni, Guido}

2017-01

Martignoni , G , Brunelli, M , Segala , D , Munari , E , Gobbo , S , Cima , L , Borze , I , Wirtanen , T, Sarhadi , V K, Atanesyan , L, Savola , S , Barzon , L, Masi , G, Fassan , M , Eble , J N , Bohling , T , Cheng , L, Delahunt , B \& Knuutila, S 2017, ' Validation of 34betaE12 immunoexpression in clear cell papillary renal cell carcinoma as a sensitive biomarker ' , Pathology , vol. 49 , no. 1 , pp. 10-18 . https://doi.org/10.1016/j.pathol.2016.05.014

http://hdl.handle.net/10138/231346

https://doi.org/10.1016/j.pathol.2016.05.014

publishedVersion

Downloaded from Helda, University of Helsinki institutional repository.

This is an electronic reprint of the original article.

This reprint may differ from the original in pagination and typographic detail.

Please cite the original version. 


\title{
Validation of 34 betaE12 immunoexpression in clear cell papillary renal cell carcinoma as a sensitive biomarker
}

\author{
Guido Martignoni $^{1,2}$, Matteo Brunelli ${ }^{1}$, Diego Segala ${ }^{2}$, Enrico Munari ${ }^{1}$, \\ Stefano Gobbo ${ }^{2}$, Luca Cima ${ }^{1}$, Ioana Borze ${ }^{3}$, Tina Wirtanen ${ }^{3}$, \\ Virinder Kaur Sarhadi ${ }^{3}$, Lilit Atanesyan ${ }^{4}$, Suvi Savola ${ }^{4}$, Luisa Barzon ${ }^{5}$, \\ Giulia Masi ${ }^{5}$, Matteo Fassan ${ }^{6}$, John N. Eble ${ }^{7}$, Tom Bohling ${ }^{3}$, Liang Cheng ${ }^{7}$, \\ Brett Delahunt $^{8}$ and Sakari KnuUtila ${ }^{3}$

\begin{abstract}
${ }^{1}$ Department of Pathology and Diagnostics, Anatomic Pathology, University and Hospital Trust of Verona, Verona, ${ }^{2}$ Pederzoli Hospital, Anatomic Pathology, Peschiera del Garda, Verona, Italy; ${ }^{3}$ Hartmann Institute and HUSLab, University of Helsinki, Department of Pathology, Helsinki, Finland; ${ }^{4}$ MRC-Holland, Amsterdam, Netherlands; ${ }^{5}$ Histology, Microbiology and Medical Biotechnologies, University of Padua, Padua, ${ }^{6}$ Department of Pathology, Anatomic Pathology, University of Padua, Padua, Italy; ${ }^{7}$ Department of Pathology and Laboratory Medicine, Indiana University School of Medicine, Indianapolis, Indiana, United States; and ${ }^{8}$ Wellington School of Medicine and Health Sciences, Department of Pathology and Molecular Medicine, University of Otago, Wellington, New Zealand
\end{abstract}

\section{Summary}

Clear cell papillary renal cell carcinoma (CCPRCC) is a recently recognised neoplasm with a broad spectrum of morphological characteristics, thus representing a challenging differential diagnosis, especially with the low malignant potential multicystic renal cell neoplasms and clear cell renal cell carcinoma. We selected 14 cases of CCPRCC with a wide spectrum of morphological features diagnosed on morphology and CK7 immunoreactivity and analysed them using a panel of immunohistochemical markers, focusing on $34 \beta \mathrm{E} 12$ and related CKs 1,5,10 and 14 and several molecular analyses such as fluorescence in situ hybridisation (FISH), array comparative genomic hybridisation $(\mathrm{aCGH}), \mathrm{VHL}$ methylation, VHL and TCEB1 sequencing and multiplex ligation-dependent probe amplification (MLPA). Twelve of $13(92 \%)$ CCPRCC tumours were positive for $34 \beta E 12$. One tumour without $3 p$ alteration by FISH revealed VHL mutation and $3 p$ deletion at aCGH; thus, it was re-classified as clear cell RCC. We concluded that: (1) immunohistochemical expression of CK7 is necessary for diagnostic purposes, but may not be sufficient to identify CCPRCC, while $34 \beta E 12$, in part due to the presence of CK14 antigen expression, can be extremely useful for the recognition of this tumour; and (2) further molecular analysis of chromosome $3 p$ should be considered to support of CCPRCC diagnosis, when FISH analysis does not evidence the common loss of chromosome 3p.

Key words: Clear cell papillary renal cell carcinoma; CK7 immunoreactivity; 34ßE12; CK14 antigen expression; FISH analysis; VHL mutation; chromosome 3 p deletion; aCGH; biomarker.

Received 27 April, accepted 22 May 2016

Available online 4 December 2016

\section{INTRODUCTION}

Clear cell papillary renal cell carcinoma (CCPRCC) is a recently recognised neoplasm that occurs in patients with end-stage renal disease and acquired cystic kidney disease as well as in otherwise normal kidneys. ${ }^{1,2}$ It is estimated that these tumours constitute up to $3 \%$ of adult renal cell carcinomas (RCCs) ${ }^{3}$ and are the fourth most common histological type of RCC. ${ }^{4}$ These tumours were originally described as being multicystic, with a prominent papillary architecture. Initial reports indicated that these tumours were composed entirely of clear cells with nuclei usually arranged in a linear fashion away from the basement membrane having a superficial resemblance to the cells of early secretory endometrium. ${ }^{1}$ Moreover, the neoplastic cells were initially reported to constantly and diffusely express cytokeratin (CK) 7 and to be predominantly negative for alpha-methylacylCoA racemase (P504S) and CD10. A characteristic genetic alteration has not yet been identified for CCPRCC; $;$,5-8 however, the majority of tumours do not show the gains of chromosome 7 and $17^{2,3,5,7}$ characteristic of papillary RCC. ${ }^{8}$ They similarly lack chromosome $3 p$ deletion and mutation or methylation of the $V H L$ gene, which characterises clear cell RCC. Rohan et al. reported a series of nine tumours showing co-expression of CAIX, HIF-1alpha, and GLUT-1 in the absence of $V H L$ gene alterations, which suggests activation of the HIF pathway by non-VHL-dependent mechanisms. ${ }^{5}$ In this study the authors concluded that these tumours were easily separable from papillary RCC with clear cell changes and clear cell RCC with focal papillary architecture, based on morphological features. Moreover, they noted that in only rare instances was the support of immunohistochemical staining an absolute requirement for differentiating between these two tumour types. ${ }^{5}$ Williamson et al. have highlighted examples of CCPRCC in which the papillary component is relatively inconspicuous, with predominance of a cystic or prominent solid or dense tubular component. They also 
showed $59 \%$ of these tumours to express CD10, especially in the cystic areas and thus to some degree mimic both low malignant potential multicystic renal cell neoplasms and conventional clear cell RCC. ${ }^{9}$ In addition to these findings, CCPRCC-like tumours have been reported in patients with or without Von Hippel-Lindau disease, underlining the difficulties in distinguishing CCPRCC from conventional clear cell RCC. ${ }^{10,11}$ More recently, Aron et al. and Deml et al. have reported a few cases of CCPRCC notably with VHL mutation $^{12,13}$ and Hakimi et al. observed TCEB 1 mutation in a series of tumours with morphological and immunohistochemical overlap with CCPRCC. ${ }^{14}$ In this study we performed a thorough immunophenotypical analysis of a total of 14 CCPRCC cases, paying special attention to the expression of $34 \beta \mathrm{E} 12$ and CK14. We also evaluated the expression of these markers in a set of tissue microarrays (TMAs) containing 150 cases of conventional clear cell RCCs. Moreover, we undertook a detailed genetic analysis of a subset of five cases with microscopic features representative of the morphological spectrum described so far in the literature in CCPRCC.

\section{MATERIALS AND METHODS}

\section{Tissue samples}

A total of 14 cases of CCPRCC were accessioned from the archives of the Department of Pathology and Diagnostics, University and Hospital Trust of Verona and Anatomic Pathology, Pederzoli Hospital of Peschiera del Garda, Verona (diagnosed from 2003 to 2012). In these cases the diagnosis was based on the presence of specific morphological features previously described in these tumours, including papillary, branching tubular, tubulo-glandular and cystic patterns. For each case, 1-12 (on average 5) paraffin-embedded tissue blocks were available. Sections $3 \mu \mathrm{m}$ thick were cut from tissue blocks and stained with haematoxylin and eosin (H\&E). The diagnosis was reviewed independently by three urological pathologists (MB, SG, GM). TMAs containing 150 cases of clear cell RCC were also used for immunostain evaluation as comparison with CCPRCC.

\section{Immunohistochemical analysis}

The immunohistochemical profile of each tumour was investigated using a panel of antibodies that consisted of: CD10 (clone 56C6, 1:10 dilution; Novocastra, USA); cytokeratin 7 (clone OV-TL 12/30, 1:400 dilution; Biogenex, USA); cytokeratin 34ßE12 (clone $34 \beta E 12,1: 40$ dilution; Dako, USA); cytokeratin 1 (clone $34 \beta \mathrm{B} 4,1: 50$ dilution; Novocastra); cytokeratin 5 (clone XM26, 1:100 dilution; Novocastra); cytokeratin 10 (clone 2HP1, 1:50 dilution; Novocastra); cytokeratin 14 (clone LL002, 1:50 dilution; Biogenex); cytokeratin AE1/AE3 (clone AE1/AE3, 1:100; Dako); parvalbumin (clone P19, 1:400 dilution; Sigma Chemical Company, USA); CAIX (polyclonal, 1:100 dilution; Abcam, UK); SLC2A1 (GLUT1; polyclonal, rabbit, 1:100 dilution; Dako); alpha-methylacyl-CoA racemase (P504S; clone 13H7, 1:50 dilution; Dako); S100A1 (clone M01, 1:800 dilution; Abnova, Taiwan); desmin (clone D33, 1:500 dilution; Dako); $\alpha$-smooth muscle actin (clone 1A4, 1:250 dilution; Dako); oestrogen receptor (1:20 dilution; Dako), progesterone receptor (clone PgR 636, 1:20 dilution; Dako); HMB45 (clone HMB45, 1:300 dilution; Dako) and cathepsin K (clone 3F9, 1:2000 dilution; Abcam). Immunoreactions were developed using a non-biotin, highly sensitive system (Envision peroxidase detection system; Dako) designed to prevent possible false-positive staining resulting from endogenous biotin present in the tissue.

\section{Protein extraction and western blot analysis}

Proteins were extracted from neoplastic tissues of six CCPRCCs and 10 clear cell RCCs. For each sample 20 serial $10 \mu \mathrm{m}$ sections were collected into an Eppendorf tube, and $150 \mu \mathrm{L}$ Cell Lysis Buffer (Cell Signaling Technology, USA) was added prior to heating at $100^{\circ} \mathrm{C}$ for $5 \mathrm{~min}$. Samples were cooled for $5 \mathrm{~min}$ on ice, centrifuged at $140,000 \times \mathrm{g}$ for $15 \mathrm{~min}$ and supernatants were transferred to a new collection tube and stored at $-20^{\circ} \mathrm{C}$. Protein quantification was performed using the Bio-Rad protein assay kit (Bio-Rad, USA) according to the manufacturer's instructions. Twenty-five $\mu \mathrm{g}$ of extracted lysates was resolved in $10 \%$ polyacrylamide SDS-PAGE gel in a BioRad Mini Protean tetra cell system at $150 \mathrm{~V}$ for $1 \mathrm{~h}$. Electrophoresed proteins were transferred into a nitrocellulose membrane at $250 \mathrm{~mA}$ for 90 min. The membranes were blocked in TBST (Tris-Buffered saline and Tween 20) plus 5\% non-fat dry milk for $1 \mathrm{~h}$ at RT with constant shaking. Subsequently, the blots were incubated overnight, washed three times with TBST and incubated with the specific secondary anti-mouse or anti-rabbit peroxidase-conjugated anti-IgG antibody (diluted 1:2000; Cell Signaling, USA). After three washes with TBST, the immunoblots were visualised with ECLplus Western Blotting Substrate (Amersham/GE Healthcare Europe, Germany). Expression levels of each marker were quantified with ImageJ (https://imagej.nih.gov/ij/index.html) densitometric analysis.

\section{Array comparative genomic hybridisation (aCGH) and data analysis}

Microdissection of formalin fixed, paraffin embedded (FFPE) kidney tumours was performed.

Genomic DNA was isolated using a QIAamp DNA mini kit (Qiagen Nordic, Finland) and quantified on the NanoDrop spectrophotometer (NanoDrop Technologies, USA). As a reference we used DNA from pooled peripheral blood leukocytes of normal males. We screened for copy number alterations in five tumours using the Agilent Human 244K array format containing 240,000 oligonucleotide probes, covering both coding and noncoding genome regions (Agilent Technologies, USA). Briefly, $1.5 \mu \mathrm{g}$ of tumour and reference DNA were digested, labelled and hybridised according to the Agilent protocols. The array images obtained after scanning (Agilent scanner G2565BA) were processed with Feature Extraction software (version 10.5), and the output data files were analysed with the Agilent Genomic Workbench. To identify copy number alterations we used the aberration detection method 2 (ADM-2) algorithm. To exclude small variances in the data we set up a custom aberration filter identifying alterations in copy number if a minimum of eight probes gained or lost were identified, with a minimum absolute average $\log$ ratio for the region being 0.5 . Regions with small copy number variations were excluded by comparing and visualising the copy number variant regions of the Genomic Workbench software tool.

\section{Fluorescence in situ hybridisation (FISH)}

FISH analysis was performed using a centromeric-specific probe for the chromosome 3 centromere (SpectrumGreen CEP3; Abbott, Italy) and a subtelomeric probe for 3p25 (SpectrumGreen 3p-LSI; Abbott) in order to evaluate $3 p$ deletion. Centromeric-specific probes for the chromosome 7 and 17 centromere were also used (SpectrumGreen, SpectrumOrange; Abbott). From the whole-tissue sections, $3 \mu \mathrm{m}$ sections were cut from paraffinembedded blocks. The paraffin was removed from the sections with two 10 min washes with xylene. After hydrating in $100 \%, 85 \%$, and $70 \%$ ethanol solutions (10 $\mathrm{min})$, rinsing in distilled water (10 min), and twice in phosphatebuffered solution ( $\mathrm{pH} 7,10 \mathrm{~min}$ each), the slides were fixed in methanol-acetic acid 3:1 for $10 \mathrm{~min}$ and air-dried. Next, the sections were treated in a $2 \mathrm{X}$ standard saline citrate solution for $15 \mathrm{~min}$ at $37^{\circ} \mathrm{C}$, dehydrated in consecutive $70 \%, 85 \%$, and $100 \%$ ethanol solution for $1 \mathrm{~min}$ each and then dried. Next, the sections were bathed in $0.1 \mathrm{mM}$ citric acid $(\mathrm{pH} 6)$ solution at $85^{\circ} \mathrm{C}$ for $1 \mathrm{~h}$ They were then dehydrated in a series of ethanol solutions and dried. The tissue was digested by applying $0.75 \mathrm{~mL}$ of pepsin (Sigma, USA) solution $(4 \mathrm{mg} / \mathrm{mL}$ in $0.9 \% \mathrm{NaCl}, \mathrm{pH} 1.5)$ to each slide and incubating in a humidified box for $30 \mathrm{~min}$ at $37^{\circ} \mathrm{C}$. Next, the slides were rinsed with distilled water for a few seconds, dehydrated in graded ethanol solutions, and dried. Centromeric probes for chromosomes 3 and the locus specific sub-telomeric probe $3 p$ were used. Each probe was diluted 1:20 in t-DenHyb-2 buffer (LiStar-FISH, Italy). ${ }^{15,16}$ Ten $\mu \mathrm{L}$ of diluted probe was applied to each slide and cover slips were placed over the slides. Denaturation was achieved by incubating the slides at $80^{\circ} \mathrm{C}$ for $10 \mathrm{~min}$ in a humidified box, and then hybridisation was carried out at $37^{\circ} \mathrm{C}$ for $16 \mathrm{~h}$. The cover slips were later removed and the slides were immersed at room temperature in $0.5 \mathrm{X} \mathrm{SSC}$ for $2 \mathrm{~min}$, in $50 \%$ formamide/1X SSC for $5 \mathrm{~min}$, and in $2 \mathrm{X}$ SSC for $2 \mathrm{~min}$. The slides were air-dried and counterstained with $10 \mathrm{~mL}$ DAPI/Antifade (DAPI in Fluorguard, $0.5 \mathrm{mg}$ / $\mathrm{ml}$; Insitus, USA). The slides were examined using an Olympus BIX-61 microscope (Olympus, Germany) with filters for SpectrumGreen, and the UV Filter for the DAPI nuclear counterstain. The signals were recorded with a 
CCD camera (Olympus Digital Camera). Fluorescent signals were evaluated as reported previously. ${ }^{2,16,17}$ Signals from 100-200 nuclei were counted, focusing only on neoplastic nuclei from the epithelial components. The control distribution of signals was assessed on non-neoplastic renal parenchyma adjacent to the tumours. The value of the ratio $(3 \mathrm{p} / 3)$ on the normal renal parenchyma $+3 \mathrm{SD}$ set to $1.03+3 \mathrm{SD} 0.05=1.19+3 \mathrm{SD}$ was used per each fluorescent score number. The percentage of neoplastic nuclei showing one, two or more than two fluorescent signals were respectively recorded as having monosomic, disomic or gains of chromosomes. Normal adjacent tissue was used as control.

\section{VHL sequencing analysis}

Five $10 \mu \mathrm{m}$ thick sections of tumour tissue were cut from FFPE blocks. DNA was extracted. Polymerase chain reaction (PCR) for $V H L$ gene analysis was performed using primer sequences as reported. ${ }^{18,19}$ Normal tissues from the same patients were used as a reference. The reaction conditions were as follows: $12.5 \mu \mathrm{L}$ of HotStart Taq PCR Master Mix (Qiagen, Germany), $10 \mathrm{pmol}$ of each primer, $100 \mathrm{ng}$ of template DNA, and distilled water up to $25 \mu \mathrm{L}$. Amplification program for all fragments, except the marker D3S666, consisted of denaturation at $95^{\circ} \mathrm{C}$ for $15 \mathrm{~min}$, then 40 cycles of denaturation at $95^{\circ} \mathrm{C}$ for $1 \mathrm{~min}$, annealing at $55^{\circ} \mathrm{C}$ for $1 \mathrm{~min}$, and extension at $72^{\circ} \mathrm{C}$ for $1 \mathrm{~min}$. The program was finished by $72^{\circ} \mathrm{C}$ incubation for $7 \mathrm{~min}$. Annealing temperature for fragment D3S666 was $58^{\circ} \mathrm{C}$. PCR products of the $V H L$ gene were purified with Montage PCR Centrifugal Filter Devices (Millipore, USA) and sequenced using a Big Dye Terminator Sequencing kit (PE/Applied Biosystems, USA). Samples were then run on an automated sequencer (ABI Prism 310; PE/Applied Biosystems) at a constant voltage of $11.3 \mathrm{kV}$ for $20 \mathrm{~min}$. PCR products of STR markers were mixed with a size marker and run on an automated sequencer (ABI Prism 310; PE/Applied Biosystems) at a constant voltage of $15 \mathrm{kV}$ for $28 \mathrm{~min}$.

Genomic DNA was isolated from three $5 \mu \mathrm{M}$ thick paraffin sections of each renal carcinoma sample using the Ex-Wax DNA Extraction Kit (Chemicon International, USA) according to the manufacturer's instructions. Bidirectional sequencing of PCR products was performed using an ABI Prism BigDye terminators v3.1 cycle sequencing kit (Applied Biosystems), and sequences were run on an Applied Biosystems 3130 Genetic Analyzer and compared with the reference sequence CCDS 2597.1. The PCR amplicon carrying the mutation was subcloned into a pGEM-T Easy vector (Promega, USA), transformed in competent DH5 $\alpha$ cells and plated onto LB agar with ampicillin and X-gal selection. Then, 12 distinct blank (white) colonies were chosen, plasmid DNA was extracted and submitted for amplification and sequencing of $V H L$ exon 3 as described above.

\section{Methylation-specific multiplex ligation-dependent probe amplification} (MS-MLPA) and CpG methylation analysis

Microdissection of tissues from the five FFPE kidney tumours was performed manually. Genomic DNA extracted from the three samples was subjected to MS-MLPA using ME001-0808-C1 and ME002-0809-B1 probemixes (MRCHolland, The Netherlands) with $20-100$ ng of DNA per sample. The standard MS-MLPA-protocol was employed. ${ }^{20}$ Both probemixes contained one specific MLPA probe for the exon 1 of $V H L$ gene that has a recognition site for a CpG methylation-sensitive endonuclease HhaI. The MS-MLPA product fragments were analysed by an ABI model 3130 capillary sequencer (Applied Biosystems, The Netherlands) using Genescan-ROX 500 size standards. As the same probemixes are intended to detect both copy number and methylation changes of the target genes simultaneously, both methylation and copy number status was analysed using Coffalyser software (MRC-Holland). The data were first normalised by dividing the peak area of a single probe by a cumulative peak area of all control probes (not degraded by HhaI). Then, the normalised peaks from the HhaI digestion reaction were compared to the normalised peaks from the undigested control reaction. Final methylation value for each sample was obtained by subtracting the background methylation values of the control samples (male and female DNA samples; Promega). The following criteria were used for determining the methylation status: 0.00-0.25 (absent), 0.25-0.50 (mild), 0.50-0.75 (moderate), and >0.75 (extensive methylation). For copy number analysis the following cut-off values were used: $<0.7$ and $>1.3$ gain. One DNA sample (labelled 8656) was excluded from the analysis due to the low amount of DNA and for another sample (labelled 10684) we were able to include only results using
ME002-0809-B1 probemix as no DNA was available for the further analysis using the ME-001-0808 mix.

\section{TCEB1 mutation analysis}

TCEB1 gene Y79 and A100 hotspots were analysed by Sanger sequencing (Cases 1-5). PCR products were purified using Agencourt AMPure XP magnetic beads (Beckman Coulter, USA) and labelled with Big Dye Terminator v3.1 (Applied Biosystems, Italy). Agencourt CleanSEQ magnetic beads (Beckman Coulter) were used for post-labelling DNA fragment purification, and sequence analysis was performed on an Applied Biosystems 3130xl Genetic Analyzer.

\section{RESULTS}

\section{Clinical and pathological findings}

The patients were nine males and five females, with a mean age of 61 years (range 46-77 years). All tumours were well circumscribed, with a mean diameter of $2.4 \mathrm{~cm}$ (range $1.2-4.0 \mathrm{~cm}$ ). All cases were pT1a. The clinical and pathological data of the cases are summarised in Table 1. Macroscopically, one tumour (Case 5) exhibited a predominantly solid and greyish appearance, while the other 13 showed a variable cystic and solid morphology (Fig. 1 and 2). No necrotic foci were seen. One tumour (Case 3) was yellow with a solid-microcystic appearance reminiscent of a conventional clear cell RCC. Microscopically, tumours showed encapsulation with differing architectural patterns as summarised in Table 2. The papillary pattern was unique in that it occurred secondarily within enlarged tubules and cysts. Other architectural patterns were also present. These consisted of branching tubules similar to benign prostatic acini, and tubulo-glandular structures of variable sizes and shape, sometimes with ill-formed lumina imparting a solid appearance. Variable sized cysts were also seen, predominantly at the periphery of the tumour. All tumour cells had clear cytoplasm with nuclei aligned circumferentially, resembling secretory endometrium (Fig. 2E-H). The nuclei of all cases were predominantly low grade (ISUP nucleolar grade 1 or 2) (Fig. 3A-D). The stroma was hyalinised although occasionally fibroleiomyomatous areas were also seen. Necrosis, mitotic activity, vascular invasion, or sarcomatoid change was not observed in any case.

\section{Immunohistochemical findings}

Immunohistochemical findings are summarised in Table 3 and Fig. 3. Half of the cases were positive for CD10, ranging from $5 \%$ to $70 \%$ of neoplastic cells, and there was diffuse positivity for CK7 in $70-100 \%$ of cells. CK AE1AE3 expression was seen in $100 \%$ of the neoplastic cells in all cases. SLC2A1 (GLUT1) and CAIX displayed variable intensity of expression in all, and in all but one case, respectively (one case not available for CAIX). Cytokeratin $34 \beta E 12$ and CK14 showed an equivalent pattern of staining as they were expressed in the same 12 cases $(12 / 14,86 \%)$; Cases 3 and 6 were completely negative for both markers (Fig. 3E-H). CK1 and CK10 were constantly negative while CK5 showed positivity in four cases (percentages variable from 10 to $80 \%$ ). Alpha-methylacyl-CoA racemase (P504S), HMB-45, cathepsin k, and oestrogen and progesterone receptors were constantly negative. S100A1 was expressed in nine of 13 cases (one case was not available), while parvalbumin showed weak immunoreactivity in $20 \%$ 
Table 1 Main clinical and pathological data of the 14 cases of clear cell papillary renal cell carcinoma

\begin{tabular}{|c|c|c|c|c|c|c|c|c|}
\hline Case no. & Sex & Age & Surgery & Diameter, $\mathrm{cm}$ & Necrosis & Grade & Follow-up, months & $\mathrm{pT}$ \\
\hline 1 & $\mathrm{~F}$ & 59 & Left nephrectomy & 2 & Absent & 2 & 48 & pT1a \\
\hline 3 & M & 55 & Enucleation & 4 & Absent & 1 & 83 & pT1a \\
\hline 4 & M & 55 & Right nephrectomy & 3 & Absent & 2 & 122 & pT1a \\
\hline 5 & $\mathrm{~F}$ & 61 & Enucleation & 3.4 & Absent & 2 & 141 & pT1a \\
\hline 6 & $\mathrm{~F}$ & 64 & Enucleation & 1.6 & Absent & 1 & 54 & pT1a \\
\hline 8 & M & 72 & Enucleation & 1.8 & Absent & 1 & 106 & pT1a \\
\hline 9 & M & 50 & Enucleation & 1.6 & Absent & 2 & 129 & pT1a \\
\hline 10 & $\mathrm{M}$ & 61 & Enucleation & 3.8 & Absent & 1 & 137 & pT1a \\
\hline 11 & $\mathrm{~F}$ & 64 & Enucleation & 3.2 & Absent & 2 & 75 & pT1a \\
\hline 12 & M & 68 & Enucleation & 2.1 & Absent & 2 & 24 & pT1a \\
\hline 13 & $\mathrm{~F}$ & 51 & Enucleation & 1.2 & Absent & 2 & 38 & pT1a \\
\hline 14 & M & 46 & Enucleation & 1.5 & Absent & 2 & 27 & pT1a \\
\hline
\end{tabular}

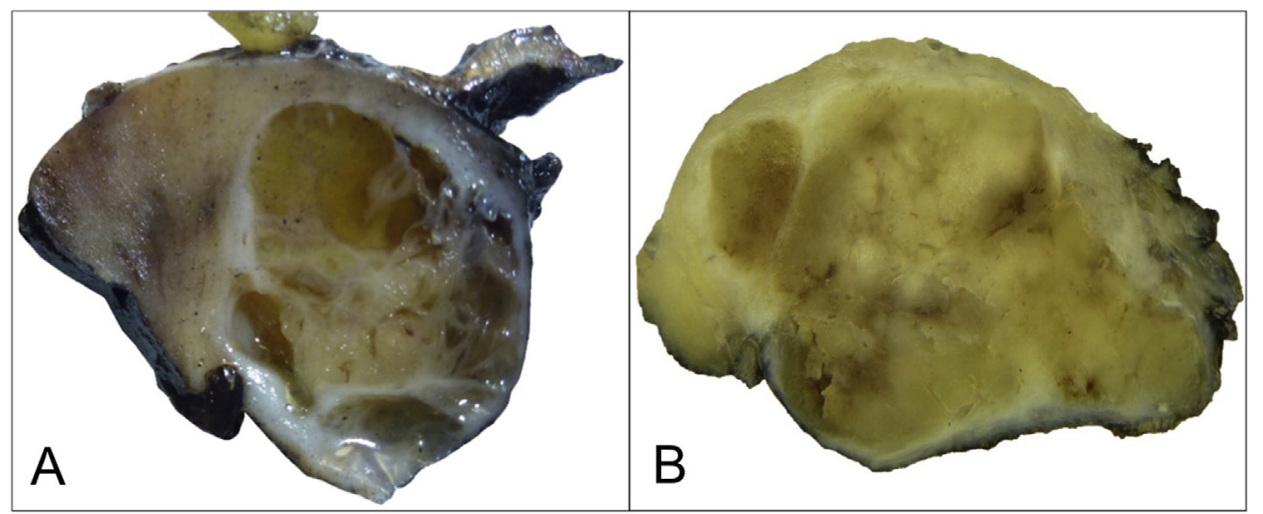

Fig. 1 Macroscopically, (A) Case 4 showed cystic and focally solid morphology, whereas (B) Case 5 exhibited a predominantly solid and white-mahogany brown appearance.

and $10 \%$ of the neoplasic cells in Cases 10 and 13, respectively. The stroma of the tumours showed focal positivity for alpha-smooth muscle actin in 12 of 14 cases, whereas there was complete negativity in the remaining two tumours. Only two of 150 clear cell RCC cases $(1 \%)$ of the TMAs stained weakly positive for $34 \beta \mathrm{E} 12$ and CK14. We previously stained the same 150 conventional clear cell RCCs for CK7 and found it positive in 36 of 150 (24\%) of the cases.

\section{Western blot results}

Two cases (Cases 2 and 4) revealed the appropriate positive band referring to CK14, respectively positive at immunohistochemistry in $60 \%$ and $40 \%$ of neoplastic cells. One case (Case 3) did not express the protein band, and was negative at immunohistochemistry. One case (Case 5) did not show evidence of the protein band but expression was observed in up to $40 \%$ of neoplastic cells at immunohistochemistry. The remaining two cases showed faint protein bands (Cases 10 and 14). Eight of 10 conventional clear cell RCCs were negative and two tumours showed a weak positive band.

\section{aCGH results}

We did not observe any gene copy number alterations in four of five cases analysed. DNA copy number changes were found in one tumour (Case 3) that showed deletions in chromosomes 3 (3p26.3q23, 3q25.1q25.2 and 3q25.32q26.2) and 6 (6q15q27) (Fig. 4, Table 4).

\section{FISH findings}

We analysed five cases by FISH.

\section{Locus specific sub-telomeric $3 p$ probe}

Single, double and three or more fluorescent signals, respectively, were shown in neoplastic epithelial nuclei as follows: Case 1, 33\%, 58\% and 9\%; Case 2, 34\%, 61\% and $5 \%$; Case 3, 31\%, 65\% and 3\%; Case 4, 35\%, 59\% and 6\%; Case $5,39 \%, 55 \%$ and $6 \%$.

\section{Centromeric chromosome 3 probe}

Single, double and three or more fluorescent signals, respectively, were shown in neoplastic epithelial nuclei as follows: Case 1, 29\%, 61\% and 10\%; Case 2, 35\%, 62\% and $3 \%$; Case $3,11 \%, 69 \%$ and $20 \%$; Case $4,44 \%, 53 \%$ and $9 \%$; Case $5,35 \%, 60 \%$ and $5 \%$. The value of the ratio of the normal renal parenchyma $+3 \mathrm{SD}$ set to $1.03+3 \mathrm{SD}$ $0.05=1.19$. The ratio was 1.02 in Case 1 (not deleted), 1.09 in Case 2 (not deleted), 1.18 in Case 3 (not deleted), 1.12 in Case 4 (not deleted) and 1.02 in Case 5 (not deleted). All five tumours showed no gains of chromosomes 7 and 17 with single signals ranging from 33 to 43 , double signals from 53 to 61 and more than two signals from 5 to 15 , respectively.

\section{VHL gene mutation}

No mutation of coding sequence of the $V H L$ gene was found in epithelial neoplastic components in four of five cases, 


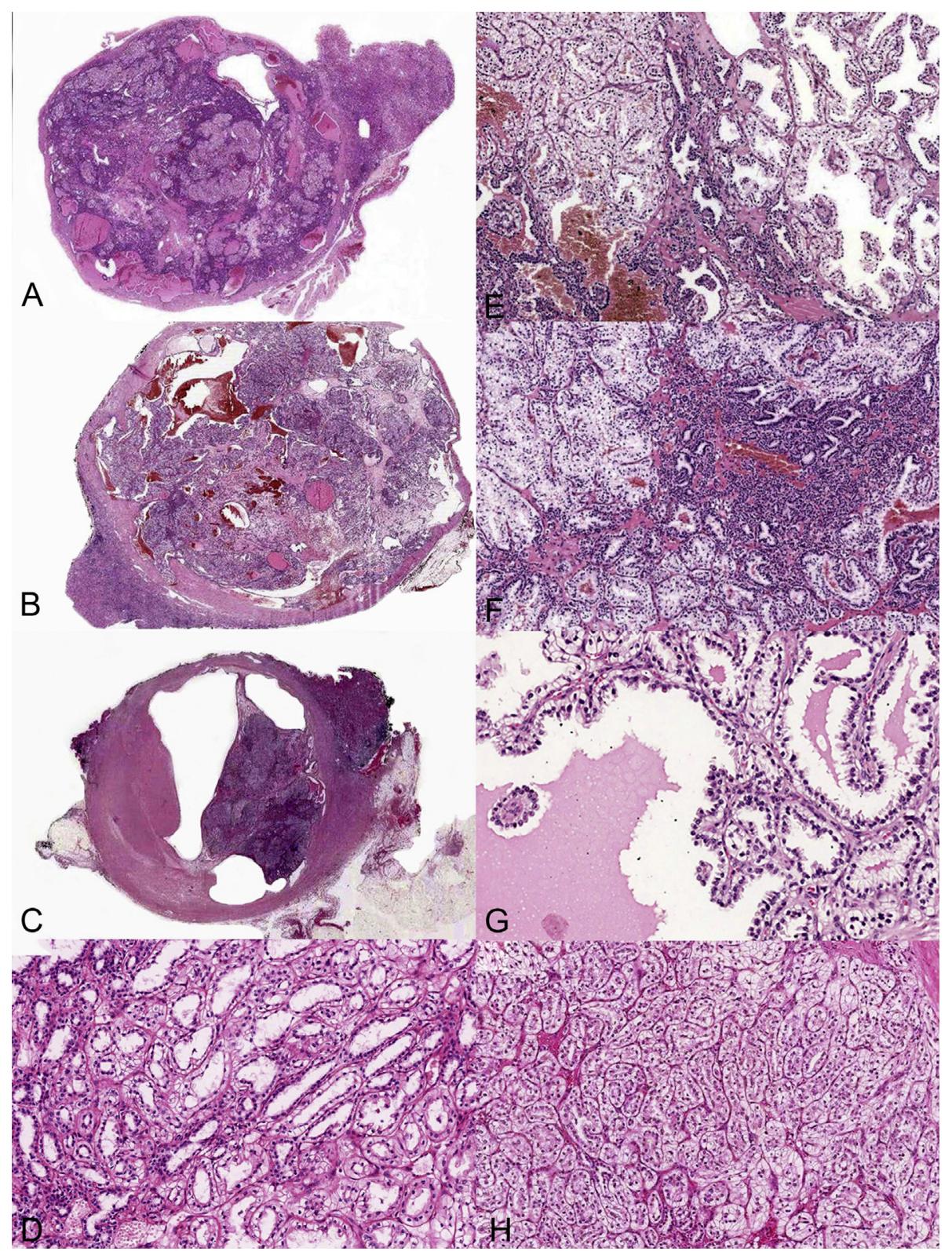

Fig. 2 The tumours show a variable cystic and solid morphology (A, Case 2; B, Case 3; C, Case 4; D, Case 5). They exhibit different architectural patterns, including tubulo-glandular structures intermixed with areas composed of branching tubules (E, Case 2; F, Case 3), cystic and papillary structures (G, Case 4) and homogeneous tubulo-glandular structures ( $\mathrm{H}$, Case 5).

whereas one tumour (Case 3) showed a deletion in exon 1 (c.213del) (Table 4).

\section{MLPA findings on methylation and copy number status of $V H L$}

MS-MLPA analysis of FFPE tissue DNA samples showed absent or mild methylation of $V H L$ gene; the copy number of $V H L$ gene was stable in all five analysed cases (Table 5).

\section{TCEB1 gene mutation}

No mutation of coding sequence of the TCEBI gene was found in any tumour.

\section{DISCUSSION}

In this study we have demonstrated that: (1) the distinction between CCPRCC and low malignant potential multicystic
RCC or conventional clear cell RCC, on the basis of morphological features, can be difficult; (2) the immunohistochemical expression of CK7 is necessary, but not sufficient to identify CCPRCC; (3) the immunoreactivity for $34 \beta \mathrm{E} 12$, in part due to the presence of CK14 in the neoplastic cells, can be extremely useful for identifying this tumour; (4) FISH can verify the presence of gains of chromosome 7 and 17 to differentiate CCPRCC from papillary RCC with clear cell changes, but it is not discriminatory when evaluating $3 p$ status in differentiating this tumour from low malignant potential multicystic RCC or conventional clear cell RCC; (5) mutational analysis is the single most reliable method to differentiate rare $\mathrm{CK} 7$ positive conventional clear cell RCCs from CCPRCC; (6) the characteristic immunoprofile, including $34 \beta \mathrm{E} 12$ expression, and genomic signature, absence of $3 p$ abnormalities, are observed in the entire morphological spectrum of CCPRCCs; (7) CCPRCC lacks 
Table 2 Frequency of different architectural pattern in the five cases selected for molecular analysis

\begin{tabular}{lllll}
\hline \multirow{2}{*}{ Case no. } & \multicolumn{4}{c}{ Architectural patterns (\%) } \\
\cline { 2 - 5 } & Papillary & Branching tubules & Tubulo-glandular & Cystic \\
\hline 1 & 60 & 0 & 10 & 30 \\
2 & 30 & 50 & 10 & 10 \\
3 & 20 & 30 & 45 & 5 \\
4 & 40 & 10 & 0 & 50 \\
5 & 10 & 0 & 90 & 0 \\
\hline
\end{tabular}

TCEB1 mutation. Although CCPRCC was initially described as a multicystic neoplasm with a prominent papillary architecture and composed of cells with clear cytoplasm, subsequent series of this tumour have shown a broader spectrum of morphological features. Aydin et al. and Williamson et al. highlighted that the papillary component is present only in $81 \%$ and $65 \%$ of cases, emphasising the branched tubular pattern, rather than the papillary pattern, as a distinctive morphological characteristic. This latter aspect, however, was often missing in tumours with a prominent cystic component. ${ }^{3,21}$ These data underscore the difficulties that may be encountered in distinguishing this tumour from papillary RCC with prominent clearing of cytoplasm, ${ }^{21}$ as well as low malignant potential multicystic clear cell and conventional clear cell RCC. Such difficulty has been clearly demonstrated by Williamson et al. who reported that 14 CCPRCCs were identified from 469 RCC resections performed from 2004 to 2006 and that the majority of these tumours were originally diagnosed as clear cell RCC. ${ }^{9}$ Their work reinforced the notion that there can be substantial morphological overlap between CCPRCC and conventional clear cell RCCs as only a single tumour with an original diagnosis of papillary RCC

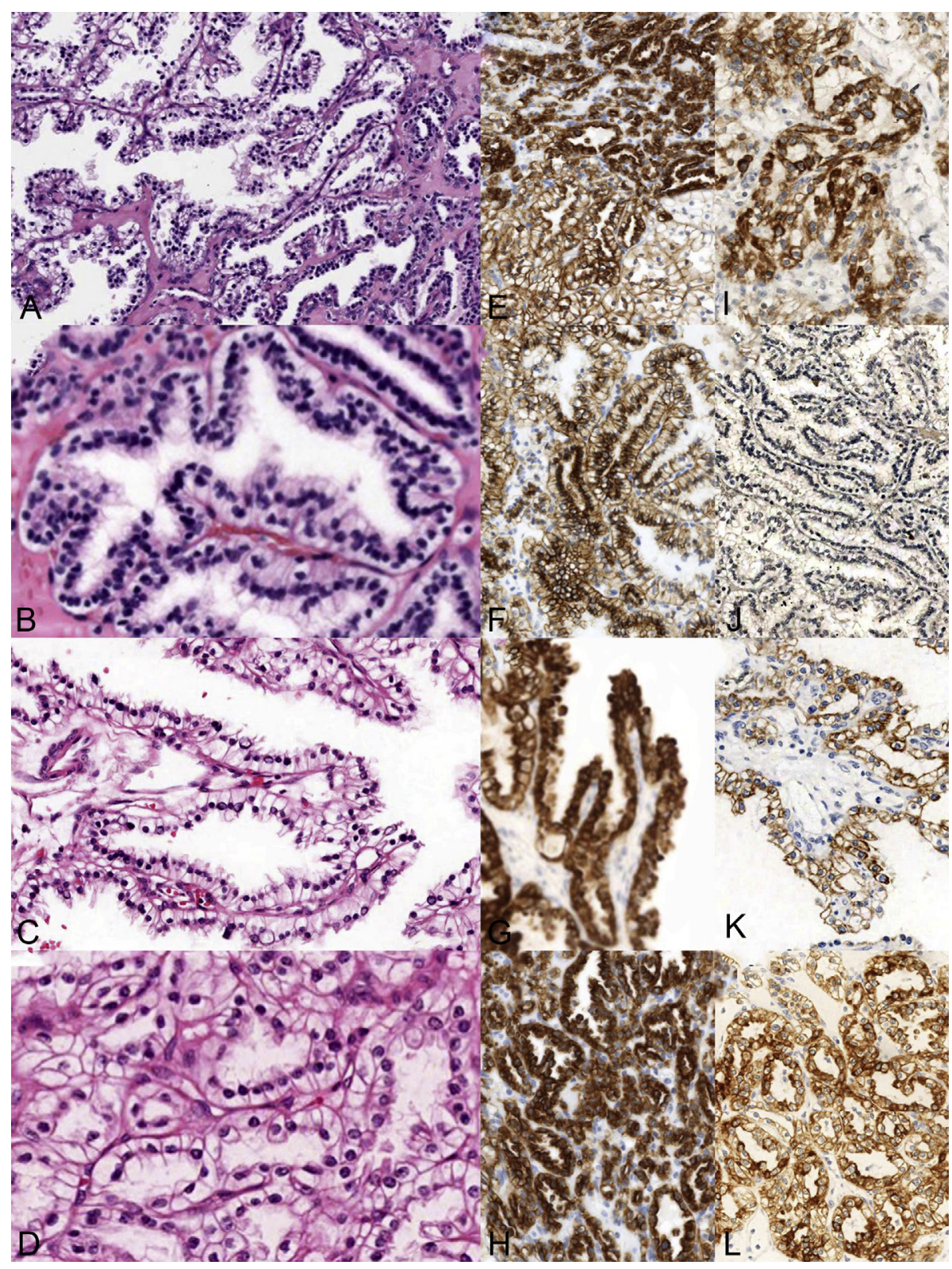

Fig. 3 The branching tubules display similar features to benign prostatic acini (A, Case 2; B, Case 3); all tumour cells show clear cytoplasm with nuclei aligned circumferentially, resembling secretory endometrium. The nuclei are predominantly low-grade (C, Case 4; D, Case 5). Diffuse expression of CK7 (E, Case 2; F, Case 3 ; G, Case 4; H, Case 5); 34ßE12 positivity (I, Case 2) and negativity (J, Case 3); immunoexpression of CK14 (K, Case 4; L, Case 5). 
Table 3 Immunohistochemical findings of the 14 cases of clear cell papillary renal cell carcinoma

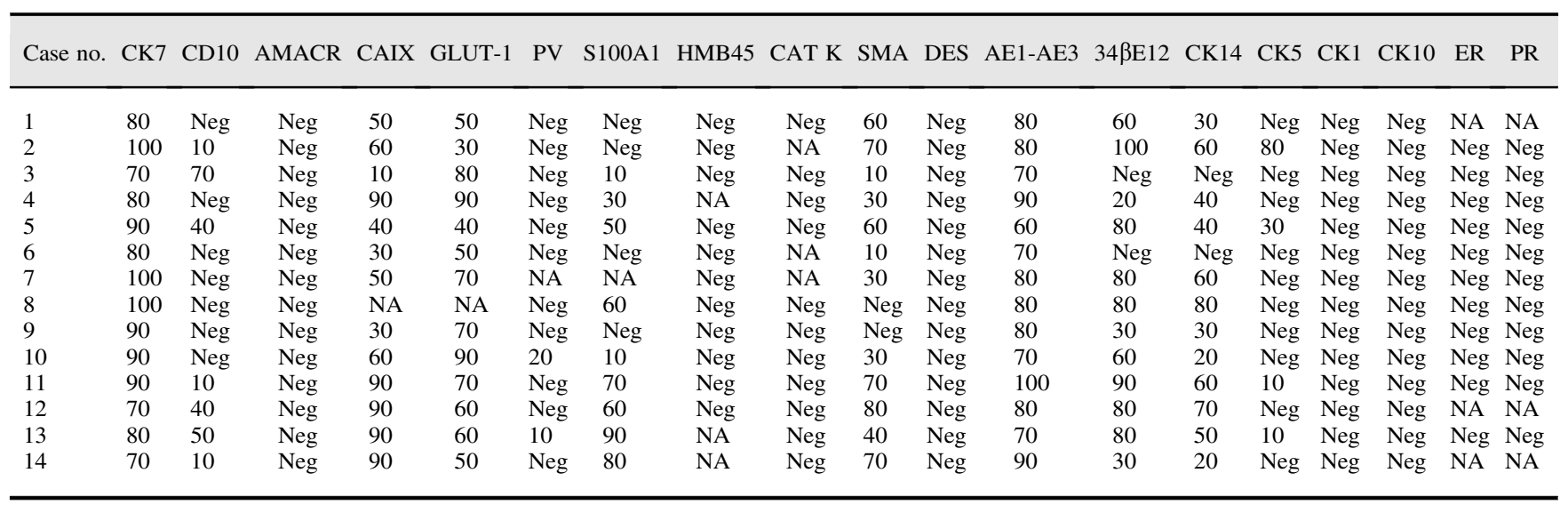

AMACR, alpha-methylacyl-CoA racemase (P504S); CAIX, carbonic anhydrase IX; CAT K, cathepsin K; CK, cytokeratin; DES, desmin; ER, oestrogen receptor; NA, not applicable; PR, progesteron receptor; PV, parvalbumin; SMA, smooth mucle actin.

Results of desmin and SMA are referred to the intratumoural stroma.

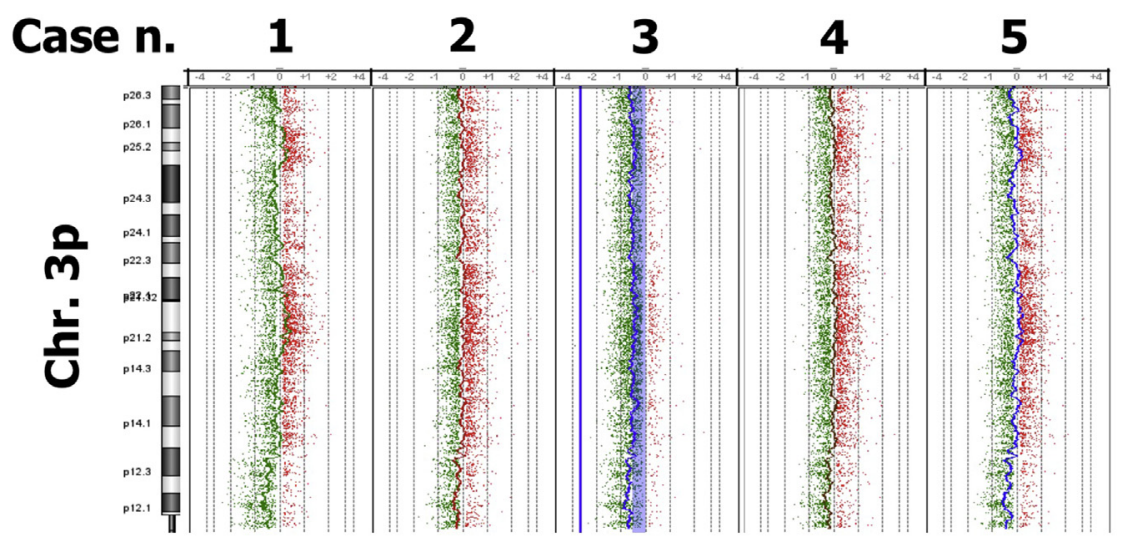

Fig. 4 Array CGH results in five cases initially diagnosed as clear cell papillary renal cell carcinoma with microscopic features representative of the morphological spectrum described so far in the literature. DNA copy number changes were found in only one tumour (Case 3 ) that presented deletions in chromosome 3 (3p26.3q23, $3 \mathrm{q} 25.1 \mathrm{q} 25.2$ and $3 \mathrm{q} 25.32 \mathrm{q} 26.2)$ and $6(6 \mathrm{q} 15 \mathrm{q} 27)$.

was reclassified, while only three were originally interpreted as multilocular cystic RCCs. Recently, CCPRCC-like tumours have been described in patients with or without von Hippel-Lindau disease unrelated to sporadic CCPRCC, highlighting once again the difficulties that one might encounter in distinguishing CCPRCC from conventional clear cell RCC. ${ }^{10}$ In support of the diagnostic difficulties mentioned above, one of the tumours (Case 3) that we selected as CCPRCC, based on morphological and immunophenotypical features (diffuse papillary architecture with a characteristic arrangement of the nuclei, as well as the presence of branched tubules and CK7 positivity), proved to be a conventional clear cell RCC with alterations of $3 p$ identified by aCGH and $V H L$ mutation. This tumour showed a strong and diffuse immunoreactivity for CK7 (70\% of the neoplastic cells), a marker currently considered as extremely useful for differentiating CCPRCC from clear cell RCC. ${ }^{2,5}$ Although the majority of clear cell RCCs lack CK7 immunoexpression, some cases have been reported to be positive for this marker. We performed a literature search encompassing a total of 391 cases of clear cell RCC and found that 44 (12\%) expressed CK7. ${ }^{22-25}$ Moreover, in our hands we have demonstrated this immunoreactivity in $24 \%$ of clear cell RCC. Finally, Williamson and Cheng have reported a group of clear cell
RCCs with borderline features of clear cell RCC, including cytokeratin 7 expression in 18 of 22 and $34 \beta E 12$ in seven of $21 .^{26}$

As we demonstrated here, this might lead to misdiagnosis, even in the presence of morphological features typical of CCPRCC. $34 \beta E 12$ is an antibody that recognises different high molecular weight cytokeratins including CK1, 5, 10 and 14 ; interestingly, we found $34 \beta \mathrm{E} 12$ to be positive in all but two cases (12/14), one of these being the CK7 positive clear cell RCC case. Of note, only in 2011 was it initially reported that $34 \beta E 12$ immunoexpression was observed in CCPRCC, and only recently Brimo et al. ${ }^{27}$ and Aron et al. ${ }^{12}$ reported additional CCPRCC with such positivity (Table 6). Aron et al. showed 41 of 42 cases with immunoexpression of $34 \beta \mathrm{E} 12 .{ }^{12}$ Rohan et al. showed seven of nine $(78 \%)$ of their CCPRCC cases to be positive for this marker; on the other hand, 34 $\beta \mathrm{E} 12$ immunostain was not detected in any of their clear cell RCC tested (0/11). In our control group accounting for 150 clear cell RCCs, only two cases (1\%) showed positivity for this marker. In order to better understand which specific cytokeratin is most responsible for the observed $34 \beta E 12$ positivity in CCPRCC, we evaluated the immunoexpression of CK1, CK5, CK10 and CK14 separately. We found that, among these molecules, CK14 followed by CK5 
Table 4 Synthesis of molecular findings of the five cases initially diagnosed as clear cell papillary renal cell carcinoma with microscopic features representative of the morphological spectrum described so far in the literature

\begin{tabular}{|c|c|c|c|c|c|c|}
\hline Case no. & aCGH & Chr $3 p$ & Ratio $3 / 3 p$ & Chr 7 & Chr 17 & VHL status \\
\hline 1 & Normal status & Disomy & 1.02 & Disomy & Disomy & WT \\
\hline 3 & $3 \mathrm{p} 26.3 \mathrm{q} 23,3 \mathrm{q} 25.1 \mathrm{q} 25.2,3 \mathrm{q} 25.32 \mathrm{q} 26.2,6 \mathrm{q} 15 \mathrm{q} 27$ & Disomy & 1.18 & Disomy & Disomy & Deletion in exon 1 (c.213del) \\
\hline 4 & Normal status & Disomy & 1.12 & Disomy & Disomy & WT \\
\hline 5 & Normal status & Disomy & 1.02 & Disomy & Disomy & WT \\
\hline
\end{tabular}

Findings from three different molecular methods investigating chromosomal imbalances: array comparative genomic hybridisation (aCGH), chromosomes $3 \mathrm{p}, 7$ and 17 status (fluorescence in situ hybridisation; FISH) and VHL status (gene sequencing).

Table 5 Methylation status and gene copy number of the five cases initially diagnosed as clear cell papillary renal cell carcinoma with microscopic features representative of the morphological spectrum described so far in the literature

\begin{tabular}{|c|c|c|c|c|}
\hline \multirow[t]{3}{*}{ Case no. } & \multicolumn{2}{|c|}{ Methylation status } & \multicolumn{2}{|c|}{ Copy number } \\
\hline & \multicolumn{4}{|c|}{ Gene (mapview position) } \\
\hline & $\begin{array}{c}\text { VHL } \\
(03-010158426)\end{array}$ & $\begin{array}{c}\text { VHL } \\
(03-010158544)\end{array}$ & $\begin{array}{c}\text { VHL } \\
(03-010158426)\end{array}$ & $\begin{array}{c}\text { VHL } \\
(03-010158544)\end{array}$ \\
\hline 1 & 0.00 & 0.00 & 1.16 & 1.05 \\
\hline 2 & 0.11 & 0.28 & 1.14 & 1.08 \\
\hline 4 & 0.00 & 0.00 & 0.99 & 1.03 \\
\hline 5 & 0.00 & 0.08 & 1.01 & 0.92 \\
\hline
\end{tabular}

Table 6 CK34ßE12 immunoexpression in clear cell papillary renal cell carcinoma

\begin{tabular}{|c|c|c|c|}
\hline Authors & $\begin{array}{c}\text { Year of } \\
\text { publication }\end{array}$ & $\begin{array}{l}\text { No. } \\
\text { cases }\end{array}$ & CK34ßE12 immunoprofiling \\
\hline Tickoo et al. & 2006 & 15 & IHC not performed \\
\hline Gobbo et al. & 2008 & 5 & CK34 $\beta E 12$ not performed \\
\hline Mai et al. & 2008 & 10 & CK34 $\beta E 12$ not performed \\
\hline Lopez et al. & 2010 & 12 & $0 / 12$ \\
\hline Aydin et al. & 2010 & 33 & CK34 $\beta E 12$ not performed \\
\hline Adam et al. & 2011 & 24 & CK34 $\beta E 12$ not performed \\
\hline Wolfe et al. & 2011 & 1 & CK34 $\beta E 12$ not performed \\
\hline Rohan et al. & 2011 & 9 & $7 / 9(78 \%)$ \\
\hline Park et al. & 2012 & 15 & CK34 $\beta E 12$ not performed \\
\hline Bhatnagar et al. & 2012 & 14 & CK34 3 E12 not performed \\
\hline Cui et al. & 2013 & 20 & CK $34 \beta E 12$ not performed \\
\hline Williamson et al. & 2013 & 34 & CK34 3 E12 not performed \\
\hline Shi et al. & 2013 & 11 & CK34 3 E12 not performed \\
\hline Fisher et al. & 2014 & 17 & CK34 $\beta E 12$ not performed \\
\hline Rao et al. & 2014 & 3 & CK34 $\beta E 12$ not performed \\
\hline Zhou et al. & 2014 & 12 & CK34ßE12 not performed \\
\hline Alexiev et al. & 2014 & 5 & $5 / 5(100 \%)$ \\
\hline Alexiev et al. & 2014 & 28 & $28 / 28(100 \%)$ \\
\hline Leroy et al. & 2014 & 42 & CK $34 \beta E 12$ not performed \\
\hline Lawrie et al. & 2014 & 17 & CK34 $\beta E 12$ not performed \\
\hline Deml et al. & 2015 & 27 & CK34 $\beta E 12$ not performed \\
\hline Yan et al. & 2015 & 6 & CK $34 \beta E 12$ not performed \\
\hline Diolombi et al. & 2015 & 58 & CK $34 \beta E 12$ not performed \\
\hline Brimo et al. & 2015 & 9 & $9 / 9(100 \%)$ \\
\hline Aron et al. & 2015 & 45 & $43 / 45(96 \%)$ \\
\hline Martignoni et al. & This study & 14 & $12 / 13(92 \%)$ \\
\hline
\end{tabular}

IHC, immunohistochemistry.

are the most highly immunoexpressed high molecular weight cytokeratins in CCPRCC; these results are more likely to be verified by western blot analysis rather than immunohistochemical analysis. We also evaluated the expression of CK14 in TMAs containing 150 cases of conventional clear cell RCC and only the two cases positive for $34 \beta E 12$ expressed CK14. Therefore, we suggest that applying $34 \beta E 12$ to the immunohistochemical panel for diagnosing CCPRCC might be useful for distinguishing the latter from conventional clear cell RCC. Although FISH analysis is an extremely valuable aid for discriminating the different RCC histotypes, this is not always the case for CCPRCC versus clear cell RCC. ${ }^{5,15}$ In fact, in the tumour that we reclassified as clear cell RCC, only aCGH and gene sequencing allowed us to detect chromosomal imbalances and VHL alteration. However, FISH analysis remains a precise tool for detection of chromosome 7 and 17 gains, thus permitting the distinction between CCPRCC and papillary RCC. Interestingly, after the reclassification of our Case 3 as clear cell RCC, we went back to the diagnostic report of this tumour where it was described as a solid and microcystic yellowish lesion typical of clear cell $\mathrm{RCC}$, which was in contrast to the gross characteristics of the other tumours in our series. Finally, Hakimi et al. ${ }^{14}$ have recently reported that at least part of the so called RCC with angioleiomyoma-like stroma ${ }^{28,29}$ which shows overlapping morphological and immunophenotypical features with CCPRCC, carries mutations in TCEB1, a gene that contributes to the $V H L$ complex to ubiquitinate hypoxiainducible factor. We confirmed their data regarding the absence of TCEB1 mutations in CCPRCC. Distinguishing CCPRCC from clear cell RCC is important, since the former has yet to be reported as having a malignant potential in cases with extensive genetic studies. As we noted above, despite having often distinctive morphological and immunohistochemical characteristics, these two entities can show overlapping features in few cases, resulting in diagnostic errors. In this study we have demonstrated that CK7 positivity and FISH evaluation for $3 p$ deletion, although 
frequently useful, might not be sufficient for distinguishing CCPRCC from conventional clear cell RCC in a minority cohort. In view of this we propose the use of $34 \beta \mathrm{E} 12$ to improve diagnostic accuracy in this differential diagnosis. and VHL mutation analysis should be considered the gold standard to evaluate cases characterised by chromosome $3 \mathrm{p}$ deletion associated with both CK7 and $34 \beta E 12$ expression.

Conflicts of interest and sources of funding: Internal Funding from Department of Pathology and Diagnostics, Anatomic Pathology, University of Verona (GM, MB, FUR 2014) has been used in part for study-related facilities. The authors state there are no conflicts of interest to disclose.

Address for correspondence: Prof Guido Martignoni, Department of Pathology and Diagnostics, Anatomic Pathology, University and Hospital Trus of Verona, P. le L. Scuro n. 10, 37134, Verona, Italy. E-mail: guido. martignoni@univr.it

\section{References}

1. Tickoo SK, dePeralta-Venturina MN, Harik LR, et al. Spectrum of epithelial neoplasms in end-stage renal disease: an experience from 66 tumor-bearing kidneys with emphasis on histologic patterns distinct from those in sporadic adult renal neoplasia. Am J Surg Pathol 2006; 30: 141-53.

2. Gobbo S, Eble JN, Grignon DJ, et al. Clear cell papillary renal cell carcinoma: a distinct histopathologic and molecular genetic entity. Am J Surg Pathol 2008; 32: 1239-45.

3. Aydin H, Chen L, Cheng L, et al. Clear cell tubulopapillary renal cell carcinoma: a study of 36 distinctive low-grade epithelial tumors of the kidney. Am J Surg Pathol 2010; 34: 1608-21.

4. Zhou H, Zheng S, Truong LD, et al. Clear cell papillary renal cell carcinoma is the fourth most common histologic type of renal cell carcinoma in 290 consecutive nephrectomies for renal cell carcinoma. Hum Pathol 2014; 45: 59-64.

5. Rohan SM, Xiao Y, Liang Y, et al. Clear-cell papillary renal cell carcinoma: molecular and immunohistochemical analysis with emphasis on the von Hippel-Lindau gene and hypoxia-inducible factor pathwayrelated proteins. Mod Pathol 2011; 24: 1207-20.

6. Wolfe A, Dobin SM, Grossmann P, et al. Clonal trisomies 7,10 and 12, normal $3 p$ and absence of VHL gene mutation in a clear cell tubulopapillary carcinoma of the kidney. Virchows Arch 2011; 459: 457-63.

7. Adam J, Couturier J, Molinie V, et al. Clear-cell papillary renal cell carcinoma: 24 cases of a distinct low-grade renal tumour and a comparative genomic hybridization array study of seven cases. Histopathology 2011; 58: 1064-71.

8. Cheng L, Williamson SR, Zhang S, et al. Understanding the molecular genetics of renal cell neoplasia: implications for diagnosis, prognosis and therapy. Expert Rev Anticancer Ther 2010; 10: 843-64.

9. Williamson SR, Eble JN, Cheng L, et al. Clear cell papillary renal cell carcinoma: differential diagnosis and extended immunohistochemical profile. Mod Pathol 2013; 26: 697-708.

10. Williamson SR, Zhang S, Eble JN, et al. Clear cell papillary renal cell carcinoma-like tumors in patients with von Hippel-Lindau disease are unrelated to sporadic clear cell papillary renal cell carcinoma. Am J Surg Pathol 2013; 37: 1131-9.

11. Petersson F, Grossmann P, Hora M, et al. Renal cell carcinoma with areas mimicking renal angiomyoadenomatous tumor/clear cell papillary renal cell carcinoma. Hum Pathol 2013; 44: 1412-20.

12. Aron M, Chang E, Herrera L, et al. Clear cell-papillary renal cell carcinoma of the kidney not associated with end-stage renal disease: clinicopathologic correlation with expanded immunophenotypic and molecular characterization of a large cohort with emphasis on relationship with renal angiomyoadenomatous tumor. Am J Surg Pathol 2015; 39: $873-88$

13. Deml KF, Schildhaus HU, Comperat E, et al. Clear cell papillary renal cell carcinoma and renal angiomyoadenomatous tumor: two variants of a morphologic, immunohistochemical, and genetic distinct entity of renal cell carcinoma. Am J Surg Pathol 2015; 39: 889-901.

14. Hakimi AA, Tickoo SK, Jacobsen A, et al. TCEB1-mutated renal cell carcinoma: a distinct genomic and morphological subtype. Mod Pathol 2015; 28: 845-53.

15. Brunelli M, Fiorentino M, Gobbo S, et al. Many facets of chromosome $3 p$ cytogenetic findings in clear cell renal carcinoma: the need for agreement in assessment FISH analysis to avoid diagnostic errors. Histol Histopathol 2011; 26: 1207-13.

16. Cossu-Rocca P, Eble JN, Delahunt B, et al. Renal mucinous tubular and spindle carcinoma lacks the gains of chromosomes 7 and 17 and losses of chromosome $\mathrm{Y}$ that are prevalent in papillary renal cell carcinoma. Mod Pathol 2006; 19: 488-93.

17. Brunelli M, Eble JN, Zhang S, et al. Metanephric adenoma lacks the gains of chromosomes 7 and 17 and loss of $\mathrm{Y}$ that are typical of papillary renal cell carcinoma and papillary adenoma. Mod Pathol 2003; 16: $1060-3$.

18. van Houwelingen KP, van Dijk BA, Hulsbergen-van de Kaa CA, et al. Prevalence of von Hippel-Lindau gene mutations in sporadic renal cell carcinoma: results from The Netherlands cohort study. BMC Cancer 2005; 5: 57.

19. Choueiri TK, Vaziri SA, Jaeger E, et al. von Hippel-Lindau gene status and response to vascular endothelial growth factor targeted therapy for metastatic clear cell renal cell carcinoma. J Urol 2008; 180: 860-5.

20. Nygren AO, Ameziane N, Duarte HM, et al. Methylation-specific MLPA (MS-MLPA): simultaneous detection of $\mathrm{CpG}$ methylation and copy number changes of up to 40 sequences. Nucleic Acids Res 2005; 33: e128.

21. Williamson SR, Halat S, Eble JN, et al. Multilocular cystic renal cell carcinoma: similarities and differences in immunoprofile compared with clear cell renal cell carcinoma. Am J Surg Pathol 2012; 36: $1425-33$.

22. Skinnider BF, Folpe AL, Hennigar RA, et al. Distribution of cytokeratins and vimentin in adult renal neoplasms and normal renal tissue: potential utility of a cytokeratin antibody panel in the differential diagnosis of renal tumors. Am J Surg Pathol 2005; 29: 747-54.

23. Pan CC, Chen PC, Ho DM. The diagnostic utility of MOC31, BerEP4 RCC marker and CD10 in the classification of renal cell carcinoma and renal oncocytoma: an immunohistochemical analysis of 328 cases. Histopathology 2004; 45: 452-9.

24. Ohta Y, Suzuki T, Shiokawa A, et al. Expression of CD10 and cytokeratins in ovarian and renal clear cell carcinoma. Int J Gynecol Pathol 2005; 24: 239-45.

25. Kim MK, Kim S. Immunohistochemical profile of common epithelial neoplasms arising in the kidney. Appl Immunohistochem Mol Morphol 2002; 10: $332-8$.

26. Williamson SR, Cheng L. Do clear cell papillary renal cell carcinomas occur in patients with von Hippel-Lindau disease? Hum Pathol 2015; 46: $340-1$.

27. Brimo F, Atallah C, Li G, et al. Cystic clear cell papillary renal cell carcinoma: is it related to multilocular clear cell cystic neoplasm of low malignant potential? Histopathology 2016; 68: 666-72.

28. Williamson SR, Cheng L, Eble JN, et al. Renal cell carcinoma with angioleiomyoma-like stroma: clinicopathological, immunohistochemical, and molecular features supporting classification as a distinct entity. Mod Pathol 2015; 28: 279-94.

29. Martignoni G, Brunelli M, Segala D, et al. Renal cell carcinoma with smooth muscle stroma lacks chromosome $3 \mathrm{p}$ and VHL alterations. Mod Pathol 2014; 27: 765-74. 